\title{
PLANET TOPERS: Planets, Tracing the Transfer, Origin, Preservation, and Evolution of their ReservoirS
}

\author{
Veronique Dehant (1), Doris Breuer (2), Philippe Claeys (3), Vinciane Debaille (4), Johan De Keyser (5), \\ Emmanuelle Javaux (6), Steven Goderis (3), Ozgur Karatekin (1), Nadine Mattielli (4), Lena Noack (1), Tilman \\ Spohn (2), Ann Carine Vandaele (5), Frank Vanhaecke (7), Tim Van Hoolst (1), Valerie Wilquet (5), and the the \\ Planet Topers group Team \\ (1) Observatoire Royal de Belgique, Bruxelles, Belgium (veronique.dehant@oma.be, 00322 3749822), (2) Deutsche Zentrum \\ fur Luft- und Raumfahrt, Berlin, Germany, (3) Vrije Universiteit Brussel, Brussels, Belgium, (4) Universite Libre de \\ Bruxelles, Brussels, Belgium, (5) Belgian Institute for Space Aeronomy, Brussels, Belgium, (6) Universite de Liege, Belgium, \\ (7) Universiteit Gent, Ghent, Belgium
}

The PLANET TOPERS (Planets, Tracing the Transfer, Origin, Preservation, and Evolution of their ReservoirS) group is an Inter-university attraction pole (IAP) addressing the question of habitability in our Solar System. Habitability is commonly understood as "the potential of an environment (past or present) to support life of any kind" (Steele et al., 2005, http://mepag.jpl.nasa.gov/reports/archive.html). Based on the only known example of Earth, the concept refers to whether environmental conditions are available that could eventually support life, even if life does not currently exist (Javaux and Dehant, 2010, Astron. Astrophys. Rev., 18, 383-416, DOI: 10.1007/s00159-010-0030-4). Life includes properties such as consuming nutrients and producing waste, the ability to reproduce and grow, pass on genetic information, evolve, and adapt to the varying conditions on a planet (Sagan, 1970, Encyclopedia Britannica, 22, 964-981). Terrestrial life requires liquid water. The stability of liquid water at the surface of a planet defines a habitable zone (HZ) around a star. In the Solar System, it stretches between Venus and Mars, but excludes these two planets. If the greenhouse effect is taken into account, the habitable zone may have included early Mars while the case for Venus is still debated. Important geodynamic processes affect the habitability conditions of a planet.

As envisaged by the group, this IAP develops and closely integrates the geophysical, geological, and biological aspects of habitability with a particular focus on Earth neighboring planets, Mars and Venus. It works in an interdisciplinary approach to understand habitability and in close collaboration with another group, the Helmholtz Alliance "Life and Planet Evolution", which has similar objectives. The dynamic processes, e.g. internal dynamo, magnetic field, atmosphere, plate tectonics, mantle convection, volcanism, thermo-tectonic evolution, meteorite impacts, and erosion, modify the planetary surface, the possibility to have liquid water, the thermal state, the energy budget and the availability of nutrients. Shortly after formation (Hadean 4.4-4.0 Ga (billion years)), evidence supports the presence of a liquid ocean and continental crust on Earth (Wilde et al., 2001, Nature, 409, 175-178), Earth may thus have been habitable very early on. The origin of life is not understood yet but the oldest putative traces of life occur in the early Archaean $(\sim 3.5 \mathrm{Ga})$. Studies of early Earth habitats documented in rock containing traces of fossil life provide information about environmental conditions suitable for life beyond Earth, as well as methodologies for their identification and analyses. The extreme values of environmental conditions in which life thrives today can also be used to characterize the "envelope" of the existence of life and the range of potential extraterrestrial habitats. The requirement of nutrients for biosynthesis, growth, and reproduction suggest that a tectonically active planet, with liquid water is required to replenish nutrients and sustain life (as currently known). These dynamic processes play a key role in the apparition and persistence of life. This contribution will focus on the highlights of the work of the IAP Planet TOPERS. 